\title{
Mobile Fleet Economic Efficiency Calculation in International Road Transport
}

\author{
Ildarkhanov Radik Fanisovich
}

Candidate of Technical Sciences, Associated Professor of Car Department, Kazan Federal University, Naberezhnye Chelny, Russian Federation E-mail: rfanis@mail.ru

\author{
Doi:10.5901/mjss.2015.v6n2s4p59
}

\begin{abstract}
Activity efficiency of economic entity in transport business considerably depends on appropriate mobile fleet choice. As a rule, reliable, having low exploitation costs and well-designed maintenance stations extensive network, trucks secure sound financial position for transport enterprises which carry out efficient organization of freight forwarding business. Mobile fleet purchase conditions are much worse than in European counties: leasing and credit rates are 3 - 4 times lower, and price itself is lower too. Our government decree which foresees disposal fee implementation makes the situation even worse. It's a serious issue and it should be solved, for under conditions of freight rates decrease, mobile fleet upgrading expenditures become more significant.
\end{abstract}

Keywords: Mobile fleet, upgrading expenditures, integrated investment, economic efficiency.

\section{Introduction}

International road transport mobile fleet manufacturers make trucks with similar technical specifications. Moreover, vehicle concordance to standard requirements of UNECE rules (there are 110 of them) and EU directives make construction parameters more similar. However, prices for vehicles by different manufacturers can vary in a substantially significant way. Consumers have issues with making right choice of mobile fleet. To solve this issue it's necessary to choose objective criterion and evaluate possible variants of vehicles according to it. Evaluation criterion must impartially assess mobile fleet efficiency. It should have meaning, meet requirements of all participants of automobile purchase and exploitation processes, and be easy and convenient for calculation, sufficient, reliable, comprehensive and replicable.

\section{Materials and methods}

Integrated investment economic efficiency assessment criterion - net current cost (NCC) meets all these requirements. Capital increase from investments is defined as a difference between total discounted cash flow and discounted investments amount. This value is net present value (NCC) and it indicates integrated effect from vehicle exploitation. NCC is calculated by formula:

$$
N C C=\sum_{n=0}^{T_{c h}} D C F_{n}-\sum_{n=0}^{T_{c h}} D I_{n},
$$

where $T s$ - vehicle service life, years; $D I_{n}$ - discounted investments of $n$ year; $n$-year numerical order.

$N C C$ is main investments economic efficiency assessment criterion. If $N C C>0$, then investments are paid off within considered period, investments are put up in right way. When $N C C<0$, project doesn't pay off. If $N C C=0$, project is neither profitable nor unprofitable.

When applied it's often not possible to define revenues from new facility exploitation. For example, how can we evaluate road freighter economy efficiency, used in manufacturing chain of own production? When it's difficult to calculate tangible benefit from using new facility, it can be evaluated according to criterion - specified discounted net expenditures $(S D N E)$, defined by formula

$$
\text { SDNE }=\left(\sum_{n=0}^{T c n} D P E_{n}+\sum_{n=0}^{T c c} D I_{n}\right) / \sum_{n=0}^{T c n} L_{\text {year }},
$$

where $D P E_{n}$ - discounted present expenditures of $n$-period; $L_{\text {year. } n}-$ annual vehicle mileage of $n$-period;

In $D P E_{n}$ - operating exploitation expenses are considered, which are defined by vehicle quality. This criterion is sum 
of nonrecurring and current expenditures for vehicle service period, put on beginning of investment period.

$$
D P E_{n}=D C \cdot \sum_{n=0}^{T_{c y}} C E_{n},
$$

where $C E_{n}$ - current expenditures of $n$-period. Current expenditures can include service production cost with deduction of amortization expenses, balance profit tax, profit tax, license acquisition expenses, trucking company allowances and training expenditures.

Investment profitability is defined by formula

$$
P I=\frac{\sum_{n=0}^{T_{c h}} D C F_{n}}{\sum_{n=0}^{T_{c h}} D I_{n}} .
$$

Investments profitability shows total discounted cash flow per 1 RUB of discounted investments. Investments are profitable, if $P I>1$. This condition is automatically performed when NCC $>0$. Condition $P I>1$ simultaneously shows, that expenditures for this project are paid off, and value PI equals multiples of payback. Naturally, within comparative analysis the biggest profitability is more preferred option.

Consumer is interested not only in pay off, but also in investment profitability which characterizes DAV. DAV discounting rate value, which corresponds to zero value NCC, i.e. it is defined by equation

$$
\sum_{n=0}^{T_{c a}} \frac{N C F_{n}}{(1+r)^{n}}-\sum_{n=0}^{T_{c s}} \frac{I_{n}}{(1+r)^{n}}=0
$$

regarding discounting rate $r$.

If discounting rate $r$ changes, NCC changes its mark from "+" to " - ", and there's such rate, under which NCC = 0 , and this rate is called DAV.

When applied equation (2.58) is solved via iteration, for this $r$ gets different values and NCC is found until condition $N C C<0$ is performed. DAV value is defined by equation

$$
D A V=r_{1}+\frac{N C C_{1}}{N C C_{1}+\left|N C C_{2}\right|} \cdot\left(r_{2}-r_{1}\right) \text {, }
$$
$N C C^{2}<0$.

where $r^{1}$ - discounting rate, under which corresponding $N C C^{1}>0 ; r^{2}$ - discounting rate, under which corresponding

We can assess DAV value according to possibility to return credit sum, compare investment profitability with alternative investments. DAV shows maximal allowable limit of discounting rate. For example, investments are performed on account of loan for $20 \%$, and $D A V=25 \%$, which means that investment profitability is bigger than bank interest and credits with interest will be returned. For investment condition DAV > "CC" (capital cost for enterprise) is needed to be performed.

Vehicle pay off can be defined by two ways: according to net cash flow without considering time factor ( $P$ payback) and discounting cash flow (CP-current pay off). To calculate payback, cash flow is added till the sum exceeds investment value. Year within which it exceeds is investment pay off period. Consumer compares obtained payback term value with alternative investments and makes decision to purchase vehicle. If consumer invested on account of credit, then payback term shouldn't exceed loan repayment term in case it will be returned only out of vehicle exploitation profits.

$P$ - pay off doesn't consider time value of money. This indicator enables to find out how much time will be needed for investment to bring in such sum of money which was spent on it without considering discounting influence. If for company pay off period matters, then this indicator can be used as "barrier". If pay off term is longer than barrier one, project will be rejected. After initial expenses indemnity, pay off ignores cash flow. This method prefers cash flows generated in first years.

Pay off indicator is not used separately, only as addition to other number indices - NCC, PI and DAV.

Investments pay off can be defined in current costs, i.e. using discounted cash flow (DCF). Pay off calculated according to DCF is called current pay off (CP - pay off). In this case time factor and "barrier rate" are considered. Naturally, current pay off is bigger than pay off calculated according to net current costs. $C P$ can be calculated by formula

$$
T O=m+\frac{\sum I-S_{m}}{P_{m+1}},
$$

where $S^{m}$ - cash flow sum for $m$ years, under which condition $S_{m}<\sum I<S_{m+1} ; P m+1$ - cash flow in (m+1) year is performed. 
We will study special features of economic efficiency calculations on actual example. Chelny Trucks Ltd is set up to provide transport services to freight cargos internationally on route «Naberezhnye Chelny - Munich (Germany) Naberezhnye Chelny». This enterprise has permission to perform international forwarding and is full member of AIRTC (Association of International Road Transport Carriers). To perform international forwarding truck KAMAZ-5460 with semitrailer Krone is bought. Truck is loaded with 20 tons of 1-st class cargo. Crew, consisting of two drivers, works on mobile fleet. Cast products are exported; roll paper for printing industry is imported. Described route will be the same for eight years.

Planned round trip duration is 384 hours. Transportation is regularly performed within a year. Market capacity is 19 round trips per year. One truck will forward 1500 tons of cargo. Rate quotations shall be negotiated.

The shortest distance in export direction:

Naberezhnye Chelny - Kazan - Moscow- Smolensk (1528 km) - Kozlovichi (Belorussia) $(679$ km) / Kukuryki Svitsko (Poland) (730 km)/ Frankfurt (Oder) - Munich (Germany) $(770$ km);

The shortest distance in import direction:

Munich - Frankfurt (Oder) (Germany) $(770$ km)/ Svitsko - Kukuryki (Poland) $(730$ km)/ Kozlovichi (Belorussia) Smolensk (679 km) - Moscow - Kazan - Naberezhnye Chelny (1528 km).

Total distance is $7414 \mathrm{~km}$, including on the territory of Germany $-1540 \mathrm{~km}$, on the territory of Poland $-1460 \mathrm{~km}$, on the territory of Belorussia - $1358 \mathrm{~km}$, on the territory of Russia - $3056 \mathrm{~km}$.

Border crossing: Smolensk, Kozlovichi, Frankfurt (Oder).

Cash flow calculation on truck exploitation is shown in chart 1 , and economy efficiency indicators calculations on truck exploitation KAMAZ-5460 with semitrailer Krone are shown in chart 2.

Thus, according to economic criteria NCC, DNE, PI, DAV, due to pay-off it's possible to objectively evaluate mobile fleet efficiency and make right choice. The method can be used while assessing efficiency of trucks bought on loan or lease. In this regard corresponding corrections are put into cash flow calculation chart. Offered method respects the interests of all international road transport participants.

According to practice and calculations, more expensive imported vehicles are not paid off within reviewed exploitation period [Fashiev, Ildarhanov \& Krahmaleva, 2004]. Some specialists explain this fact by low competitive ability of home forwarders. Competition on international road transport services market has considerably become more strained. It's especially noticeable under conditions of economy pace and foreign trade activity decrease in many counties. Competitive ability of international forwarders is known to depend on many factors.

Chart 2.1: Cash flow calculation from truck exploitation, RUB thousands.

\begin{tabular}{|l|c|c|c|c|c|c|c|c|c|}
\hline \multirow{2}{*}{ Indicator } & \multicolumn{7}{|c|}{ Years } \\
\hline & 0 & 1 & 2 & 3 & 4 & 5 & 6 & 7 & 8 \\
\hline 1 & 2 & 3 & 4 & 5 & 6 & 7 & 8 & 9 & 10 \\
\hline 1.Investments & & & & & & & & & \\
\hline 1.1.Truck & 1100 & & & & & & & & \\
\hline 1.1.0.VAT on truck & 198 & & & & & & & & \\
\hline 1.2.Semitrailer & 400 & & & & & & & & \\
\hline 1.2.0.VAT on semitrailer & 72 & & & & & & & & \\
\hline 1.3. Recording tachometer & 21 & & & & & & & & \\
\hline 1.3.0.VAT on recording tachometer & 3 & & & & & & & & \\
\hline 1.4.Communication facilities & 5 & & & & & & & & \\
\hline 1.4.0.VAT on communication facilities & 0,9 & & & & & & & & \\
\hline 1.6. Working assets & 346 & & & & & & & & \\
\hline 1.7.VAT on working assets & 62 & & & & & & & & \\
\hline 1.8.Expenses on gaining license, vehicle permission & 6,2 & 1,2 & 1,2 & 1,2 & 1,2 & 1,2 & 1,2 & 1,2 & 1,2 \\
\hline 1.9. Entry cost to AIRTC & 77 & & & & & & & & \\
\hline 1.10. Infrastructure expenses & 0 & & & & & & & & \\
\hline 1.11.Training expenses & 7 & & & & & 7 & & & \\
\hline 1.12.Other nonrecurring expenses & 10 & & & & & & & & \\
\hline Total investments & 1973 & & & & & & & & \\
\hline 2. Profits. Expenses. Taxes. & & & & & & & & & \\
\hline 2.1. Round trips quantity & & 19 & 19 & 19 & 19 & 18 & 18 & 17 & 17 \\
\hline
\end{tabular}




\begin{tabular}{|l|c|c|c|c|c|c|c|c|c|}
\hline 2.2.Rate for 1 round trip & & 280 & 280 & 280 & 280 & 280 & 280 & 280 & 280 \\
\hline 2.3. Operating income without VAT & & 5320 & 5320 & 5320 & 5320 & 5040 & 5040 & 4760 & 4760 \\
\hline 2.4.VAT (0\% from operating income) gained & & 0 & 0 & 0 & 0 & 0 & 0 & 0 & 0 \\
\hline 2.5. Services production cost & & 4774 & 4800 & 4829 & 4859 & 4492 & 4485 & 4339 & 4375 \\
\hline 2.5.1.Fuel expenses & & 1539 & 1570 & 1601 & 1634 & 1578 & 1610 & 1551 & 1582 \\
\hline 2.5.1.0.VAT & & 277 & 282 & 288 & 294 & 284 & 289 & 279 & 284 \\
\hline 2.5.2. Lube and technical fluids expenses & & 90 & 92 & 94 & 96 & 92 & 94 & 91 & 93 \\
\hline 2.5.2.0.VAT & & 16 & 16 & 16 & 17 & 16 & 17 & 16 & 16 \\
\hline 2.5.3.Tires expenses & & 142 & 142 & 142 & 142 & 134 & 134 & 127 & 127 \\
\hline 2.5.3.0.VAT & & 25 & 25 & 25 & 25 & 24 & 24 & 22 & 22 \\
\hline
\end{tabular}

Chart extension 2.1

\begin{tabular}{|c|c|c|c|c|c|c|c|c|c|}
\hline 1 & 2 & 3 & 4 & 5 & 6 & 7 & 8 & 9 & 10 \\
\hline 2.5.4. Maintenance and repair expenses & & 181 & 184 & 188 & 192 & 185 & 189 & 182 & 186 \\
\hline 2.5.4.0.VAT & & 32 & 33 & 33 & 34 & 33 & 34 & 32 & 33 \\
\hline 2.5.5. Drivers salary & & 480 & 480 & 480 & 480 & 480 & 480 & 480 & 480 \\
\hline 2.5.6. Social security contributions & & 127 & 127 & 127 & 127 & 127 & 127 & 127 & 127 \\
\hline 2.5.7. Carrier services expenses & & 532 & 532 & 532 & 532 & 504 & 504 & 476 & 476 \\
\hline \multicolumn{10}{|l|}{ 2.5.8. Rental payments } \\
\hline \multicolumn{10}{|l|}{ 2.5.8.0.VAT } \\
\hline 2.5.9. "Green card" insurance & & 16 & 16 & 16 & 16 & 16 & 16 & 16 & 16 \\
\hline 2.5.10.Medical insurance & & 4 & 4 & 4 & 4 & 4 & 4 & 4 & 4 \\
\hline 2.5.11. Carnet TIR purchase expenses & & 61 & 61 & 61 & 61 & 58 & 58 & 55 & 55 \\
\hline 2.5.11.0.VAT & & 11 & 11 & 11 & 11 & 10 & 10 & 9 & 9 \\
\hline 2.5.12.Transit permission expenses & & 9 & 9 & 9 & 9 & 9 & 9 & 8 & 8 \\
\hline 2.5.12.0.VAT & & 2 & 2 & 2 & 2 & 2 & 2 & 2 & 2 \\
\hline 2.5.13. International passports and visas expenses & & 4 & 2 & 2 & 2 & 2 & 4 & 2 & 20 \\
\hline 2.5.14. Truck amortization & & 263 & 263 & 263 & 263 & 46 & 0 & 0 & 0 \\
\hline 2.5.15.Semitrailer amortization & & 33 & 33 & 33 & 33 & 31 & 31 & 29 & 29 \\
\hline 2.5.15.1. Recording tachometer amortization & & 3 & 3 & 3 & 3 & 3 & 3 & 3 & 3 \\
\hline 2.5.15.2.Communication facilities amortization & & 0,6 & 0,6 & 0,6 & 0,6 & 0,6 & 0,6 & 0,6 & 0,6 \\
\hline 2.5.16. Road charges & & 79 & 79 & 79 & 79 & 75 & 75 & 71 & 71 \\
\hline 2.5.17.CMR expenses & & 1 & 1 & 1 & 1 & 1 & 1 & 1 & 1 \\
\hline 2.5.17.0.VAT & & 0,25 & 0,25 & 0,25 & 0,25 & 0,24 & 0,24 & 0,22 & 0,22 \\
\hline 2.5.18. Speed diagram expenses & & 0,38 & 0,38 & 0,38 & 0,38 & 0,36 & 0,36 & 0,34 & 0,34 \\
\hline 2.5.18.0.VAT & & 0,06 & 0,06 & 0,06 & 0,06 & 0,06 & 0,06 & 0,06 & 0,06 \\
\hline 2.5.19. Frontier charges & & 40 & 40 & 40 & 40 & 38 & 38 & 36 & 36 \\
\hline 2.5.20. Daily subsistence for drivers & & 129 & 129 & 129 & 129 & 122 & 122 & 116 & 116 \\
\hline 2.5.21. Lodging allowance for drivers & & 407 & 407 & 407 & 407 & 385 & 385 & 364 & 364 \\
\hline 2.5.21.0. VAT & & 13 & 13 & 13 & 13 & 12 & 12 & 12 & 12 \\
\hline 2.5.22.Communication expenses & & 25 & 25 & 25 & 25 & 25 & 25 & 25 & 25 \\
\hline 2.5.22.0.VAT & & 4,5 & 4,5 & 4,5 & 4,5 & 4,5 & 4,5 & 4,5 & 4,5 \\
\hline
\end{tabular}

Chart extension 2.1

\begin{tabular}{|l|c|c|c|c|c|c|c|c|c|}
\hline \multicolumn{1}{|c|}{1} & 2 & 3 & 4 & 5 & 6 & 7 & 8 & 9 & 10 \\
\hline 2.5.23. Optional insurance (MOD insurance) & & 37 & 28 & 20 & 12 & 4 & 3 & 3 & 3 \\
\hline 2.5.24. Membership fees in AIRTC & & 8 & 8 & 8 & 8 & 8 & 8 & 8 & 8 \\
\hline 2.5.25. Representational expenses & & 10 & 10 & 10 & 10 & 10 & 10 & 9 & 9 \\
\hline 2.5.26. Additional charges & & 528 & 528 & 528 & 528 & 528 & 528 & 528 & 528 \\
\hline 2.5.26.0.VAT & & 47 & 47 & 47 & 47 & 47 & 47 & 47 & 47 \\
\hline 2.5.27. Tax for vehicle owner & & & & & & & & & \\
\hline 2.5.28. Credits interests & & & & & & & & & \\
\hline 2.5.29. Land fee & & & & & & & & & \\
\hline 2.5.30. Lease payments & & 545 & 519 & 490 & 460 & 547 & 554 & 420 & 384 \\
\hline 2.6.Services operating profit & & & & & & & & & \\
\hline 2.7. Income and expenses due to assets disposal & & & & & & & & \\
\hline
\end{tabular}




\begin{tabular}{|l|c|c|c|c|c|c|c|c|c|}
\hline 2.8. Non-operational income and expenses & & & & & & & & & \\
\hline 3. Balance profit & & $\mathbf{5 4 5}$ & $\mathbf{5 1 9}$ & $\mathbf{4 9 0}$ & $\mathbf{4 6 0}$ & $\mathbf{5 4 7}$ & $\mathbf{5 5 4}$ & $\mathbf{4 2 0}$ & $\mathbf{3 8 4}$ \\
\hline 4. Balance profit taxes & & 37 & 31 & 25 & 19 & 13 & 11 & 10 & 9 \\
\hline 4.1. Property tax & & 37 & 31 & 25 & 19 & 13 & 11 & 10 & 9 \\
\hline 5. Taxable income & & 507 & 487 & 464 & 441 & 533 & 543 & 410 & 374 \\
\hline 6. Business tax & & 121 & 116 & 111 & 105 & 128 & 130 & 98 & 89 \\
\hline 7. Net profit & & 385 & 370 & 353 & 335 & 405 & 412 & 311 & 284 \\
\hline Cash flows correction & & & & & & & & & \\
\hline 8. Current assets change & & 0 & 0 & 0 & 0 & 18 & 0 & 18 & 309 \\
\hline 9. Credits interests & & & & & & & & & \\
\hline 10. Depreciation allocations & & 300 & 300 & 300 & 300 & 81 & 34 & 33 & 33 \\
\hline 11. Depreciable value of working assets & & & & & & & & & 419 \\
\hline 12.Net cash flow NCF & -1973 & 684 & 669 & 652 & 634 & 496 & 446 & 361 & 1045 \\
\hline 13. Discounting coefficient (DC) & & & & & & & & & \\
\hline DC within r $=70 \%$ & 1 & 0,58 & 0,34 & 0,20 & 0,12 & 0,07 & 0,04 & 0,02 & 0,01 \\
\hline 14. Discounting cash flow (DCF) & & & & & & & & & \\
\hline
\end{tabular}

Chart extension 2.1

\begin{tabular}{|l|c|c|c|c|c|c|c|c|c|}
\hline \multicolumn{1}{|c|}{1} & 2 & 3 & 4 & 5 & 6 & 7 & 8 & 9 & 10 \\
\hline DCF within $r=70 \%$ & -1973 & 402 & 231 & 133 & 76 & 34 & 18 & 8 & 14 \\
\hline 15.DCF increasing within $r=70 \%$ & -1973 & -1571 & -1339 & -1206 & -1130 & -1095 & -1077 & -1068 & -1054 \\
\hline 16.DC within $r=20 \%$ & 1 & 0,83 & 0,69 & 0,58 & 0,48 & 0,4 & 0,33 & 0,28 & 0,23 \\
\hline 17. DCF within $r=20 \%$ & -1973 & 568 & 461 & 378 & 304 & 198 & 147 & 101 & 240 \\
\hline 18. DCF increasing within $r=20 \%$ & -1973 & -1405 & -943 & -565 & -261 & -62 & 85 & 186 & 426 \\
\hline 19.Net expenses & 1973 & 4635 & 4650 & 4667 & 4685 & 4561 & 4593 & 4416 & 4443 \\
\hline 20. DC within $r=20 \%$ & 1 & 0,83 & 0,69 & 0,58 & 0,48 & 0,4 & 0,33 & 0,28 & 0,23 \\
\hline 21. Net discounting expenses (NDE) & 1973 & 3847 & 3208 & 2707 & 2249 & 1824 & 1515 & 1236 & 1021 \\
\hline 22. NDE increasing within $r=20 \%$ & 1973 & 5821 & 9030 & 11737 & 13986 & 15811 & 17326 & 18563 & 19585 \\
\hline
\end{tabular}

Chart 2.2: Economic efficiency indicators from truck exploitation

\begin{tabular}{|l|c|}
\hline Economic efficiency indicators & Value \\
\hline 1. NCC, RUB thousands. & 426 \\
\hline 2. SNDE, RUB/km & 18 \\
\hline 3. Investments profitability & 1,22 \\
\hline 4. Internal pay off coefficient, \% & 41,53 \\
\hline 5. Current pay off term, years & 5,3 \\
\hline 6. Investments, RUB thousands. & 1973 \\
\hline
\end{tabular}

\section{Conclusion}

Main participants of transport business, which compete with home enterprises at our market, are forwarders from Poland and Baltic countries. They use modern facilities more efficiently and earn approx. 10-12 thousand euro per month due to high turnover of trucks. Most part of our forwarders can hardly reach 7 thousand euro [Online resource].In European companies annual mean truck mileage exceeds 140 thousand km, and Russian ones is within $100-110$ thousand km.

Mobile fleet purchase conditions are much worse than in European counties: leasing and credit rates are $3-4$ times lower, and price itself is lower too. Our government decree which foresees disposal fee implementation makes the situation even worse. It's a serious issue and it should be solved, for under conditions of freight rates decrease, mobile fleet upgrading expenditures become more significant. Moreover, many of our forwarders consider heavy tax burden to be reason of their low competitive ability.

It's good that majority of international forwarders has got used to work and withstand difficulties. Even in this situation our leading enterprises are actively developing. Such conclusion is related to mobile fleet upgrading too. Within 2013 Russian international forwarders have acquired approx. 3 thousand of new trucks made by foreign manufacturers [Online resource]. 


\section{References}

[Online] Available: http//www.asmap.ru. Reference data: 15.06.2014.

H.A.Fashiev, R.F. Ildarhanov, A.V. Krahmaleva (2004) Mobile fleet economic efficiency assessment in international road transport. Automobile operating company, 2, 10-14. 